\title{
AUTOMATIC ENHANCEMENT OF DIGITAL IMAGES USING CUBIC BÉZIER CURVE AND FOURIER TRANSFORMATION
}

\author{
Khurshid Asgharl, Ghulam Gilanie ${ }^{2}$, Mubbashar Saddique $^{3}$, Zulfiqar Habib ${ }^{4}$ \\ ${ }^{1,2,3,4}$ Department of Computer Science, COMSATS Institute of Information Technology, Lahore, Pakistan
}

Email:khasghar@ue.edu.pk¹, gilanie@ ciitlahore.edu.pk², mubashar.chaudary@gmail.com³ drzhabib@ciitlahore.edu.pk ${ }^{4}$

\begin{abstract}
Image enhancement is one of the most important and visually appealing areas of digital image processing and computer vision. It is considered as a very important part of pre-processing for the development of industrial, security, forensic, medical, and many other applications. Although significant efforts were devoted in the past, a completely automatic image enhancement for all types of input and applications is still a big challenge in the domain of image processing. In this paper, we have proposed a multi-disciplinary approach to enhance the image automatically by intensity transformation in the spatial domain through cubic Bézier curve on images filtered with low and high pass Butterworth in the frequency domain. Results are promising when compared with the existing state-of-the-arts techniques for both visually and quantitatively, which proved that the proposed method can ensure better contrast enhancement.
\end{abstract}

Keywords: Image Enhancement, Cubic Bézier Curve, Fourier Transformation

\subsection{INTRODUCTION}

Digital image processing plays a vital role in automation of industrial applications, security surveillance, activities recognition, tracking, robotics, and many others. Image enhancement is one of the most important and visually appealing area of digital image processing and computer vision $[1,2]$. It is usually considered as part of pre-processing for most of the applications development. Although the process of image enhancement is considered problem oriented and specific to the application, however its automation is necessary to minimize the human involvement during the execution of industrial projects. Efforts were devoted in the past, but a completely automatic image enhancement suitable for all types of images: dark, bright, backlighted, low contrast, and high contrast, with no loss of the region of interests (ROIs), is still an open research issue. Image enhancement is achieved either by increasing the contrast of an image or to make prominent the hidden details in an image [3]. Due to the increase in grey levels difference [1,4], the enhanced image looks better than original one.

Researchers have been developing various intensity transformation, interpolation or approximation methods [5, 6] for image enhancement such as Histogram Equalization (HE) [7], Bézier Curve for Contrast Enhancement (BCCE) [8], and Adaptive Neighborhood Enhancement (ANE) [9], etc. HE is simple and automatic, however it does not preserve the image brightness [10,11]. Adaptive Histogram Equalization (AHE) usually gives visually pleasing results but at the cost of intrinsic imbalance and discontinuity in the structure of image due to the process of transformations independently in small slices [12]. Therefore, the results cannot be used reliably in many applications like forensic analysis and diagnosis of very small medical symptoms. Approximation for image enhancement in both the high and low frequency domain individually better results. Park and Krishna [8] proposed a cubic spline based method BCCE for the contrast enhancement of all types of images. It works well on some types of images but enhancement in some other types is not significant. Recently in [21], a Gaussian mixture model based scheme was used to enhance the contrast, however results are demonstrated for gray scale images and only one type of image is considered.

In this paper, we have proposed a spline based intensity transformation method on the frequency domain to enhance the contrast of all types of colored images. The results generated by our proposed method of image enhancement were compared both visually, and quantitatively with respect to Entropy, Mean Square Error (MSE) or Mean Square Difference (MSD), Peak Enhanced to Original Image Ratio (PEOIR), and Signal to 
Noise Ratio (SNR) [13] with other state-of-the-art methods of image enhancement, i.e., HE and BCCE. The remaining part of this paper is organized as follows. Section 2 describes the proposed approach. Section 3 describes the image enhancement evaluation parameters. Results are discussed in Section 4 followed by conclusion and future work in Section 5.

\subsection{PROPOSED METHOD}

Our methodology of image enhancement is based on the multi-disciplinary approach and completed in three major steps. First, image is transformed into low and high frequency domain followed with determining the types of image. Then spline is used for intensity approximation by using its control points according to the type of image. Low frequency is related to slowly varying intensity and separated by using a low pass filter. High frequency is caused by sharp transitions in intensity, and represents edge and noise. We have separated the high frequency using a high pass filter. After approximation of gray levels of both high and low pass images using cubic Bézier curve, the results are merged into single one, which yields a promising enhanced image. Detail is given in following sub-sections.

\subsection{Cubic Bézier curve}

Cubic Bézier curve is a parametric polynomial function frequently used in computer graphics and CAD/CAM applications for approximation [14] and interpolation of data [2]. The Bézier curve is extensively used to model data in the form of smooth curve [15]. To enhance the contrast of an image, a cubic Bézier curve was used in [8]. The cubic Bézier curve is defined in (1) and is used to model the contrast enhancement problem in different scenarios depending on scenes or image types. Desired shape of the curve for contrast enhancement may be obtained by setting the control points of the cubic Bézier function

$$
\boldsymbol{P}(t)=(1-t)^{3} \boldsymbol{P}_{0}+3 t(1-t)^{2} \boldsymbol{P}_{1}+3 t^{2}(1-t) \boldsymbol{P}_{2}+t^{3} \boldsymbol{P}_{3}
$$

where $\boldsymbol{P}_{i}, i=0,1,2,3$, are control points in 2D vector form as shown in Fig. 1 and 2.

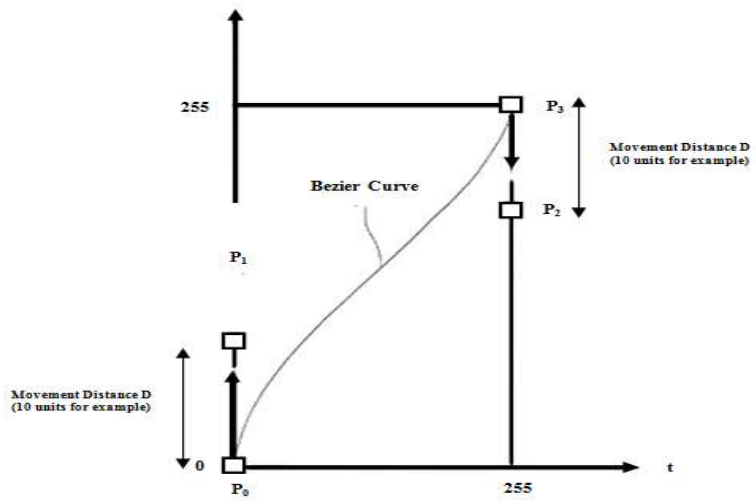

Fig. 1 Control points adjustment sample according to movement distance D [8]

\subsection{Image in frequency domain}

Normally the image $f(x, y)$ is converted into frequency domain $F(u, v)$ for analysis and processing [16]. Frequency domain allows operations on the frequency content of an image. High frequency content such as edges and other important information can easily be enhanced because edges and sharp transitions (e.g. noise) in 
an image contribute significantly to high frequency content of Fourier transform. Low frequency contents are responsible to the general appearance of the image over smooth areas [4]. Different types of filters are used to separate the high and low frequencies in the frequency domain. As described in [17], use of frequency domain instead of spatial domain is better approach to avoid unwanted changes during contrast enhancement process. Therefore, for a given image of size $M \times N$, we have used following high and low pass Butterworth filters, respectively [18]:

$$
H(u, v)=\frac{1}{1+\left[D_{0} / D(u, v)\right]^{2 n}}, H(u, v)=\frac{1}{1+\left[D(u, v) / D_{0}\right]^{2 n}}
$$

where $D_{0}$ is the radius of the circle to cut off frequency, $n$ is order of the filter and $D(u, v)$ is the distance from the point $(u, v)$ to the origin of the frequency plane and is calculated by

$$
D(u, v)=\sqrt{\left(u-\frac{M}{2}\right)^{2}+\left(v-\frac{N}{2}\right)^{2}}
$$

In $[2,4]$, it was found that, when a low pass filter is applied with radius 5,15, 30, 80 and 230 pixels, it represents the $92 \%, 94.6 \%, 96.4 \%, 98,99.5 \%$ image detail respectively. This is because the low pass filter has $93 \%$ to $96 \%$ of spectrum power, whereas the high pass filter has $4 \%$ to $7 \%$ of spectrum power that contains the edge information. We investigated 30 subjects for radius selection for low pass and high pass filters and concluded that radius of 20 for high pass filter and 100 for low pass filter produced the best results.
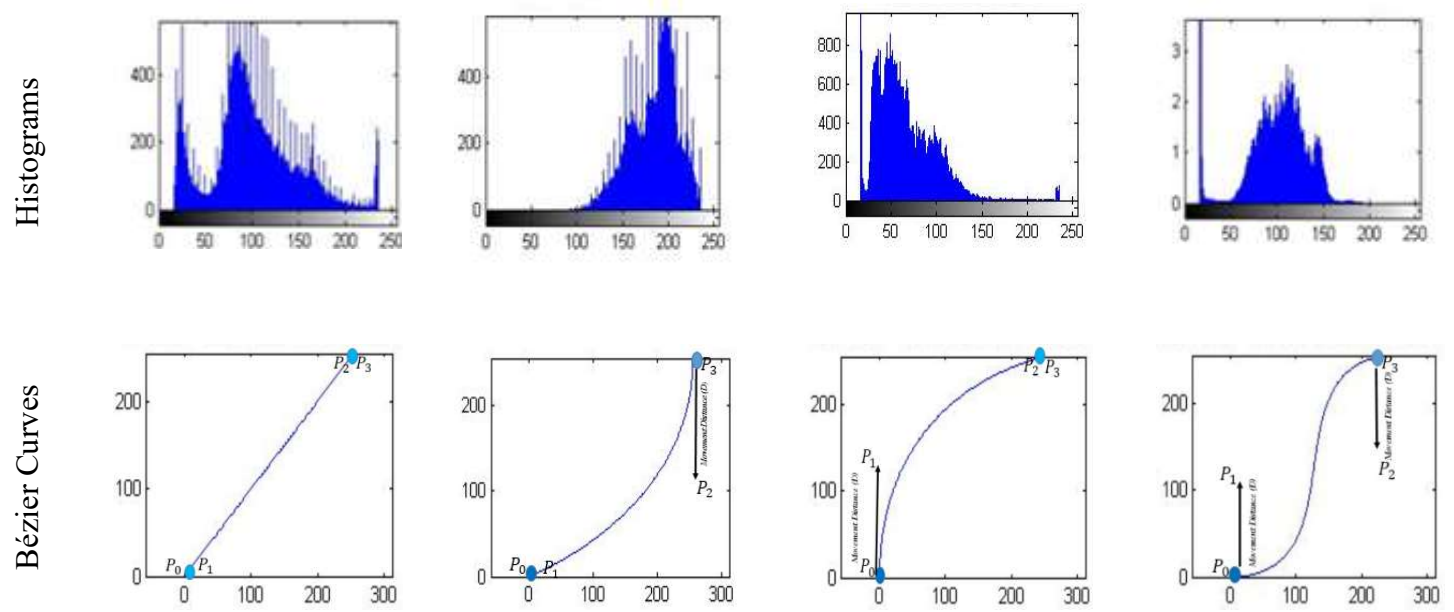

Type

(a) High Contrast

(b) Bright

(c) Dark

(d) Low Contrast

Fig. 2: Proposed positions of Bézier control points according to the types of images [8]

\subsection{Algorithm}

In this section, an automatic contrast enhancement method is proposed that performs effectively on all types of images. 


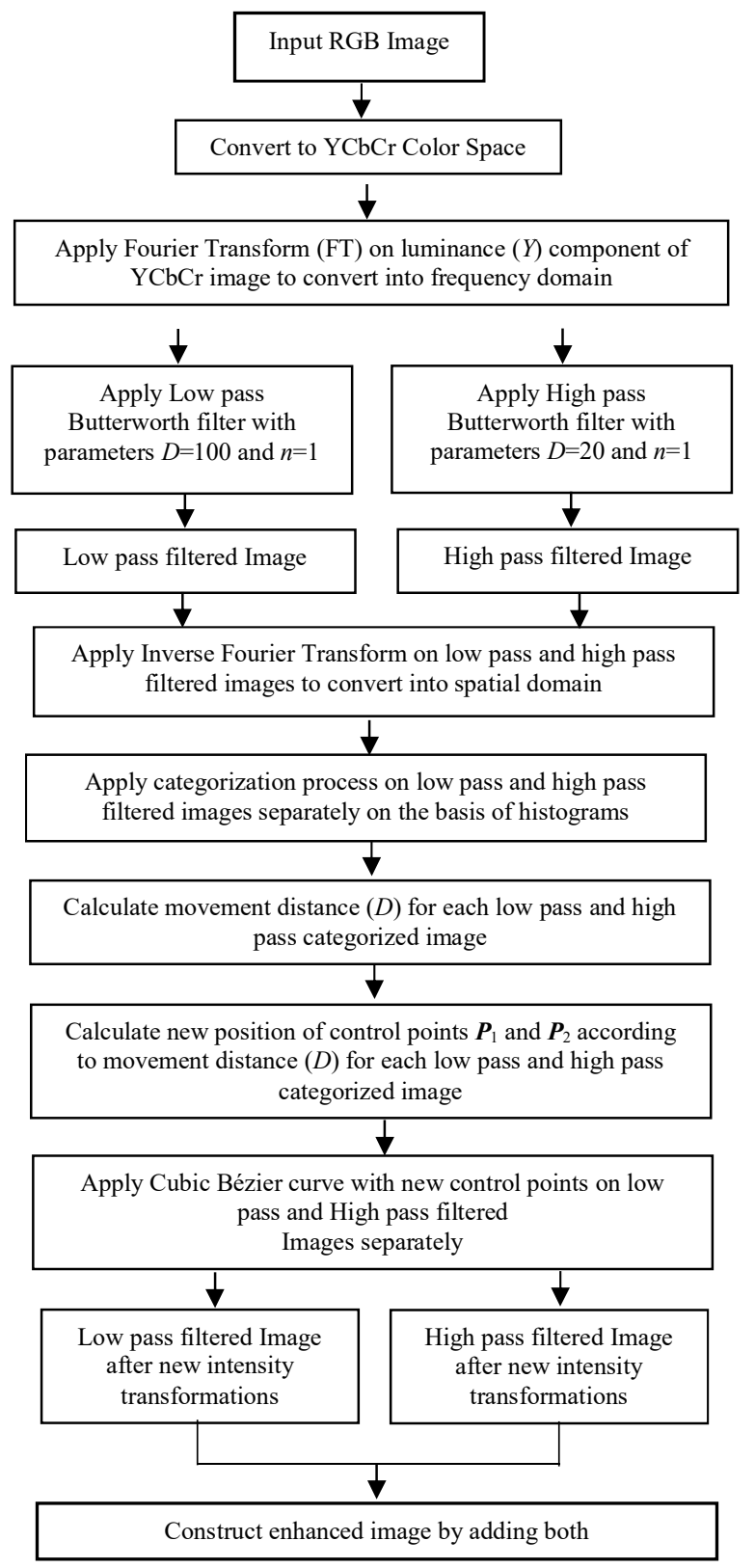

Fig. 3: Main steps of the proposed method

For our proposed method, we point out that contrast can be enhanced without significance noise amplification by applying cubic Bézier curve on images filtered using Fourier transformation. In short, our proposed method is completed in following steps:

i. $\quad$ Convert given $\mathrm{RGB}$ image into $\mathrm{YCbCr}$ color space.

ii. Extract luminance channel $Y$ from $\mathrm{YCbCr}$.

iii. Convert $Y$ from spatial domain to frequency domain using Fourier Transformation (FT).

iv. Apply Butterworth filters (low pass with $D_{0}=100$ and high pass with $D_{0}=20$ ) using $n=1$ respectively, to compute low and high pass filtered images.

v. Apply inverse FT on both low and high pass versions. 
vi. Calculate histogram of each low and high pass filtered image to categorize the image as dark or bright or low contrast or high contrast [8].

vii. Calculate movement distance $(D)$ using

$$
D=\left(2^{(N-1)}\right) * T_{c},
$$

where $T_{c}$ is calculated from

$$
T_{c}=N /\left(L_{\max }-L_{\min }+1\right)
$$

$L_{\max }$ and $L_{\min }$ are pixels with maximum and minimum luminance values of $Y$ respectively, and $N$ is the total number of pixels with frequencies greater than zero.

viii. As shown in Fig. 1, calculate new position of control points $\boldsymbol{P}_{1}$ and $\boldsymbol{P}_{2}$, according to the calculated movement distance $D$, the end points $\boldsymbol{P}_{0}$ and $\boldsymbol{P}_{3}$ remained fixed at $(0,0)$ and $(255,255)$, respectively. Calculation of middle control points for all possible types of images are illustrated as: a) over dark images: $\boldsymbol{P}_{1}=\boldsymbol{P}_{0}+(0, D), \boldsymbol{P}_{2}=\boldsymbol{P}_{3} .$, b) over bright images: $\boldsymbol{P}_{2}=\boldsymbol{P}_{3}-(0, D), \boldsymbol{P}_{1}=\boldsymbol{P}_{0}$., c) back light images: $\boldsymbol{P}_{1}=\boldsymbol{P}_{0}+(0, D), \boldsymbol{P}_{2}=\boldsymbol{P}_{3}-(0, D)$. d) low contrast images: $\boldsymbol{P}_{1}=\boldsymbol{P}_{0}+(D, 0)$, $\boldsymbol{P}_{2}=\boldsymbol{P}_{3}-(D, 0)$.

ix. Apply cubic Bézier function using control points obtained through the above process on both low pass and high pass filtered images to compute new histograms for mapping of new luminance values.

x. Finally add both low and high pass versions of previous step.

Top down approach of the proposed method is shown in Fig. 3.

\subsection{IMAGE CONTRAST ENHANCEMENT EVALUATION PARAMETERS}

Image enhancement is revolutionary approach to image processing. Contrast enhancement is a complex task as several parameters are to be considered to evaluate the performance of contrast enhancement techniques [13]. The performance evaluation of contrast enhancement depends on various factors such as entropy, signal to noise ratio, etc.

Existing studies have introduced different contrast enhancement evaluation parameters [13, 18]. Our proposed method evaluation is based on the following parameters.

\subsection{Measure of Entropy (ME)}

This metric measures the randomness of the texture of an image. The entropy is calculated by using Shannon's entropy theorem [19]. High entropy value means the image has high contrast. Entropy is calculated as

$$
M E=-\sum_{i=1}^{m} \sum_{j=1}^{n} p(i, j) \log _{2}(p(i, j))
$$

where $p$ stands for the histogram count of an image.

\subsection{Peak Enhanced to Original Image Ratio (PEOIR)}

Mean Squared Difference $(M S D)$ /Mean Square Error $(M S E)$ and Peak Enhanced to Original Image Ratio $(P E O I R)$ are commonly used measures to check the quality of images. These are calculated as

$$
M S D=\frac{1}{m n} \sum_{i=1}^{m} \sum_{j=1}^{n}\left(I_{e}-I_{o}\right)^{2}
$$




$$
P E O I R=10 \log _{10} \frac{\left(I_{\max }\right)^{2}}{M S D},
$$

where $I_{\max }$ is the maximum intensity of enhanced image. $I_{e}$ and $I_{o}$ are enhanced and original image intensities. In order to get a high-quality contrast enhanced image, the intensity difference between both enhanced and original images must be very high. Hence, the quality of processed image will be higher for high values of MSD, meaning to say that there is a large difference between the intensities of the enhanced and the original image. Therefore, PEOIR should be small for high values of MSD.

\subsection{Signal to Noise Ratio (SNR)}

The measure of ratio of the signal power to the noise power commonly known as SNR measures the foreground signals strength relative to the background noise. The SNR is calculated by

$$
S N R d b=\frac{10 \log _{10}\left(P_{\text {Signals }}\right)}{P_{\text {Noise }}},
$$

where $P_{\text {Signals }}$ and $P_{\text {Noise }}$ are mean $(\mu)$ and standard deviation $(\sigma)$ of image, respectively.

\subsection{RESULTS AND DISCUSSIONS}

The dataset used for experimentation and comparison is shown in Fig. 3. The statistical analysis is based on the outcomes of the evaluation parameters described in Section 3 [13]. Based on above mentioned parameters, Table 1 shows the comparison between proposed and other methods, i.e., HE and BCCE.

The high contrast of the image means the high value of MSE/MSD. In our case, MSE/MSD is significantly higher than HE and BCCE for different types of images. The low value of PEOIR depicts high contrast of the image. Table 1 depicts the Quantitative comparison of the proposed method and the state-of-the-art methods. As shown from the results of Table 1, the proposed method produced lower values of PEOIR as compared to the $\mathrm{HE}$ and BCCE for the same images. From Table 1 SNR values of the proposed method is significantly higher than HE and BCCE. Therefore, the overall enhancement of all types of images is improved with our proposed method. Fig. 5 provides the visual comparison of the results along with their respective histograms.

We have observed the following issues in the state-of-the-art methods. The HE made the image over bright by adding additive noise as shown in Fig. 5(a). The BCCE did not enhanced the high contrast image as shown in Fig. 5(b) although it needs some enrichment in gray levels, while the proposed method as shown in Fig. 5(c) enhanced the image properly by intelligently contributing gray levels as indicated by histogram in Fig. 5(f). In case of bright image as shown in Fig. 5(g) and its histogram is shown in Fig. 5(j), the HE made the image over bright by adding noise. The BCCE enhanced the contrast of the image as shown in Fig. 5(h). It is evident from its histogram in Fig. 5(k), that BCCE did not enhance the image properly. As such, discontinuity is observed due to less contribution of dark and bright shades of gray levels in the resultant image. On the other hand, the proposed method enhanced the image and assured the contribution of maximum gray levels as shown in Fig. 5(i) and its histogram in Fig. 5(1). Generally, in either of the case, the HE attempts to flat the histogram, causing the image to become noisy, especially in case of low and high contrast images. Since control points for Bézier curve are calculated according to the type of image in proposed method, therefore the contrast of resulting images becomes better than $\mathrm{HE}$ and $\mathrm{BCC}$ due to the suitable frequency of all gray levels. 


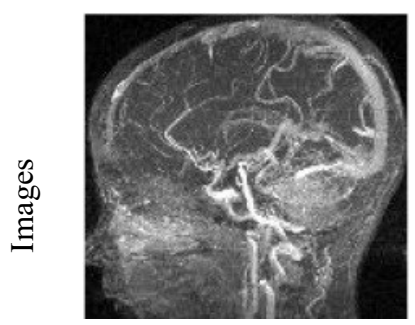

(a)

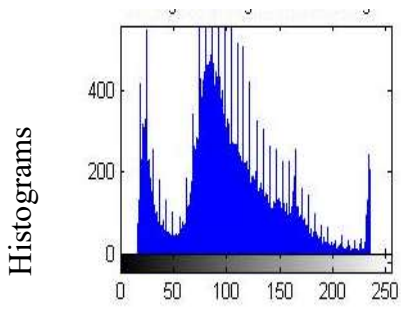

(e)

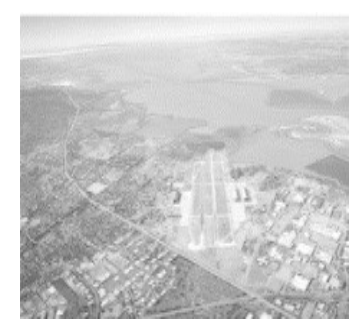

(b)

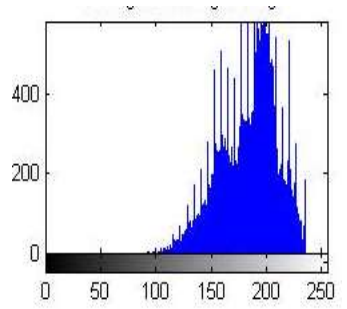

(f)

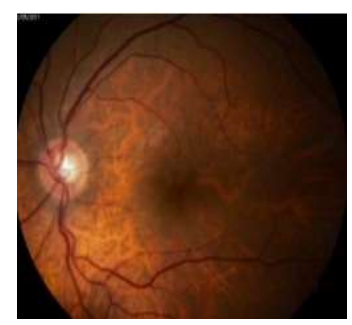

(c)

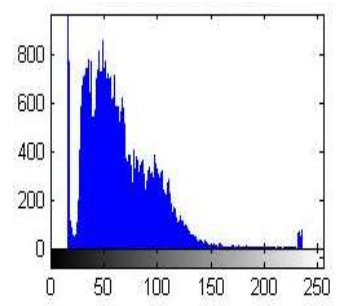

(g)

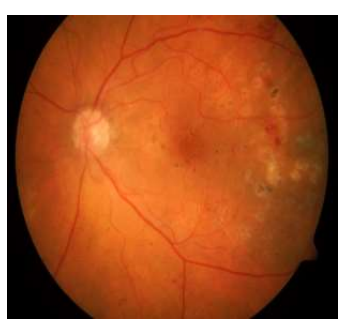

(d)

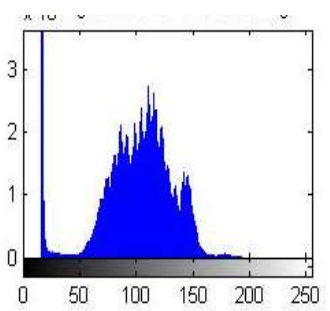

(h)

空 High Contrast

Bright

Dark

Low Contrast

Fig. 4: Dataset used for comparison of proposed method with HE [7] and BCCE [8]

\subsection{CONCLUSION AND FUTURE WORK}

Image enhancement is an essential feature in most of the image processing methods allied with specific image processing applications. The effectiveness of the existing methods depends significantly on the types of acquired images and nature of applications. Since image acquisition methods never produce same type of images even in a very controlled environment due to the dependency on many factors. As such, a comprehensive method of image enhancement is required to pre-process and normalize all types of images for applications in the area of image processing, computer vision, and robotics. Our proposed method automatically recognizes all types of images and enhances the contrast intelligently. We have performed both visual and quantitative comparison of our proposed method, with a very popular Histogram Equalization (HE) and a cubic spline based method Bézier Curve for Contrast Enhancement (BCCE). From the experimental results, we note that the proposed method provides better contrast enhancement than the existing state-of-the-arts methods, and offer an easy way to remove noises than $\mathrm{HE}$ and BCCE. Histogram specification is also sometimes very effective for the enhancement but it is a manual process, therefore its automation by using cubic Bézier curve is our future research direction. 
Table 1 Comparison between proposed method and state-of-the-art methods (HE [7], and BCCE [8])

\begin{tabular}{|c|c|c|c|c|c|c|c|c|c|c|c|c|c|c|}
\hline \multirow[b]{2}{*}{ 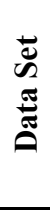 } & \multirow{2}{*}{ 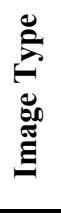 } & \multicolumn{4}{|c|}{ Entropy } & \multicolumn{3}{|c|}{$M S E / M S D$} & \multicolumn{3}{|c|}{ PEOIR } & \multicolumn{3}{|c|}{$S N R$} \\
\hline & & 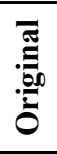 & $\underline{\underline{x}}$ & ک્ર & 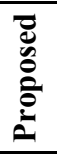 & 武 & ب્ & 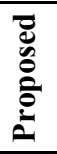 & 필 & ب̂ & 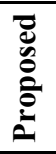 & 空 & ب̂ & 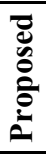 \\
\hline 1 & 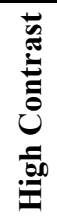 & $\stackrel{\text { }}{\sim}$ & $\hat{\tilde{n}}$ & $\stackrel{\overbrace{}}{i}$ & 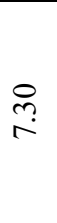 & $\frac{\infty}{n} \underset{n}{n}$ & 0 & $\begin{array}{l}n \\
\stackrel{\infty}{\infty} \\
\stackrel{\infty}{+}\end{array}$ & 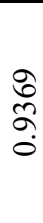 & $\begin{array}{l}\stackrel{8}{\circ} \\
\stackrel{0}{\circ}\end{array}$ & 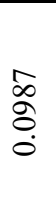 & $\begin{array}{l}\bar{\sigma} \\
\dot{+} \\
\dot{+}\end{array}$ & 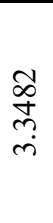 & $\begin{array}{c}\tilde{\delta} \\
\tilde{\infty} \\
+ \\
+\end{array}$ \\
\hline 2 & 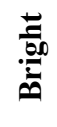 & in & $\begin{array}{l}\text { Dे } \\
\text { in }\end{array}$ & $\stackrel{0}{\longrightarrow}$ & $\stackrel{\text { ָे }}{r}$ & 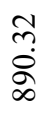 & $\begin{array}{l}\stackrel{0}{\otimes} \\
\dot{0}\end{array}$ & $\underset{\substack{\infty \\
\sim}}{\stackrel{\infty}{*}}$ & $\begin{array}{l}\text { సे } \\
\text { ते } \\
\text { ֻे }\end{array}$ & $\begin{array}{l}\hat{f} \\
\text { ò } \\
\stackrel{0}{0}\end{array}$ & 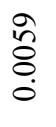 & $\begin{array}{l}\text { ปे } \\
\text { ㅎ }\end{array}$ & $\begin{array}{l}\infty \\
\infty \\
\& \\
\wp \\
\dot{m}\end{array}$ & $\begin{array}{l}\stackrel{8}{8} \\
\infty \\
\infty\end{array}$ \\
\hline 3 & ثَّ & $\stackrel{8}{:}$ & $\stackrel{2}{i}$ & $\stackrel{m}{r}$ & $\stackrel{m}{r}$ & $\ddot{0}$ & $\begin{array}{l}n \\
\tilde{D} \\
n\end{array}$ & $\begin{array}{l}\text { İ } \\
\text { J }\end{array}$ & 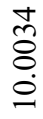 & $\begin{array}{l}\frac{1}{8} \\
\stackrel{8}{0} \\
\stackrel{0}{0}\end{array}$ & $\begin{array}{l}\text { ¿े } \\
\text { } \\
\stackrel{0}{0}\end{array}$ & 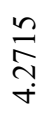 & $\begin{array}{l}\stackrel{\infty}{\circ} \\
\stackrel{\circ}{+} \\
\stackrel{+}{2}\end{array}$ & $\begin{array}{l}\vec{a} \\
\text { mे }\end{array}$ \\
\hline 4 & 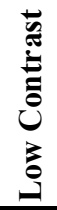 & f̊ & $\begin{array}{l}n \\
\ddot{n} \\
i\end{array}$ & $\begin{array}{l}\infty \\
\infty \\
\leftrightarrow \\
0\end{array}$ & $\stackrel{\infty}{\widehat{\sigma}}$ & 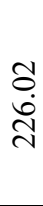 & $\stackrel{\circ}{\circ}$ & $\begin{array}{l}\ddot{\sim} \\
\ddot{n}\end{array}$ & 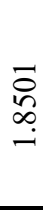 & $\begin{array}{l}\vec{J} \\
\stackrel{\text { ஸ̣ }}{0}\end{array}$ & 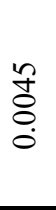 & $\underset{\dot{\sim}}{\stackrel{f}{f}}$ & $\begin{array}{l}\frac{2}{\hat{a}} \\
\tilde{n}\end{array}$ & 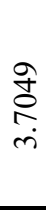 \\
\hline
\end{tabular}

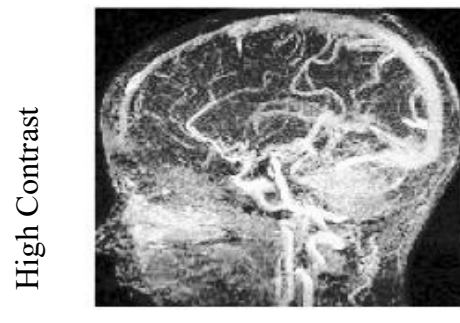

(a)

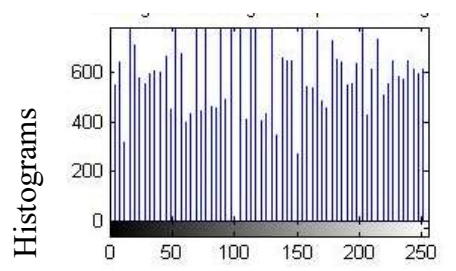

(d)

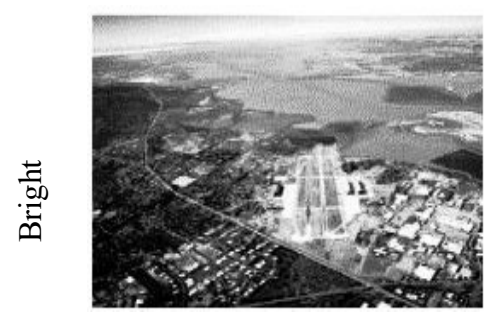

(g)

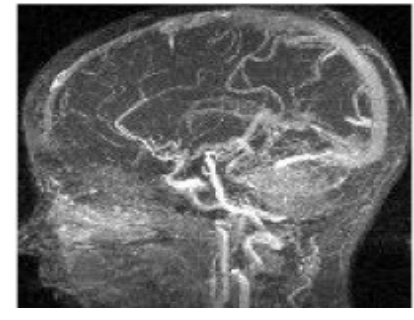

(b)

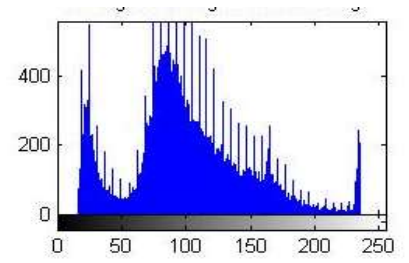

(e)

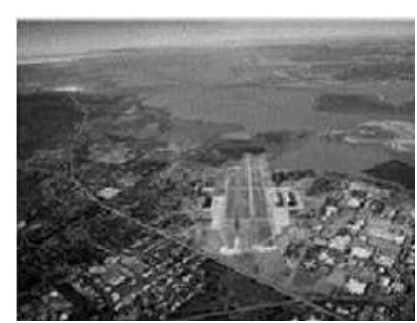

(h)

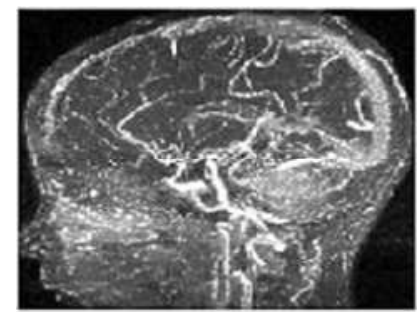

(c)

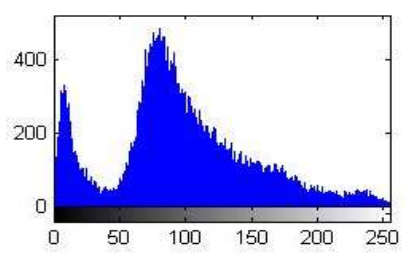

(f)

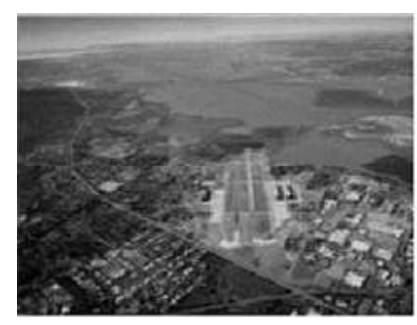

(i) 


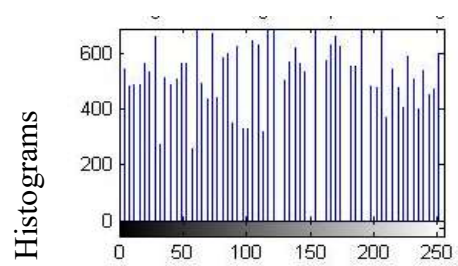

(j)

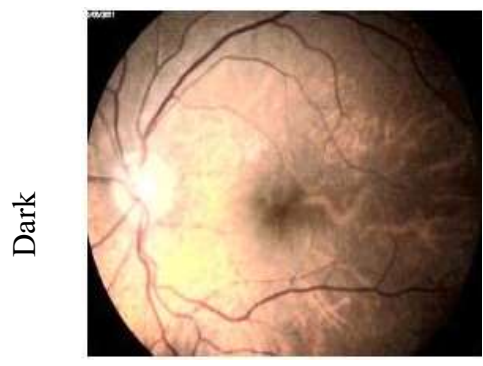

(m)

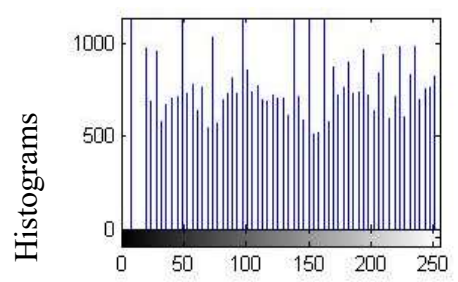

(p)

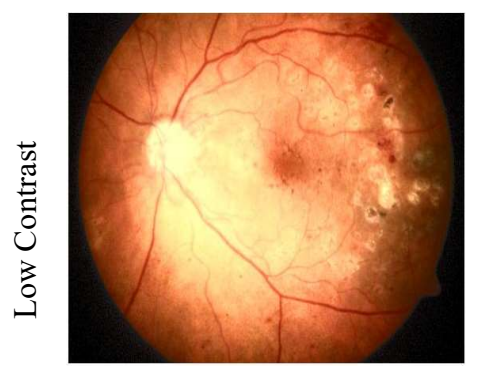

(s)

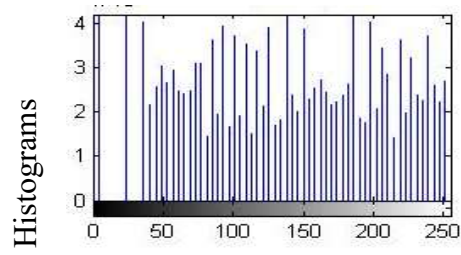

(v)

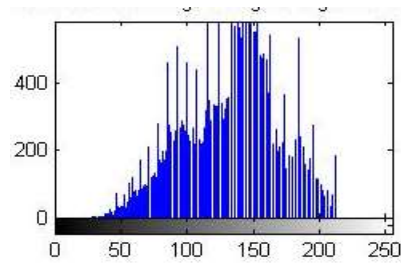

$(\mathrm{k})$

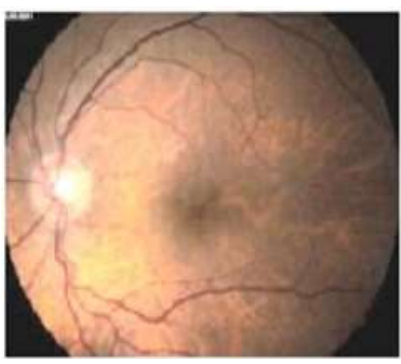

(n)

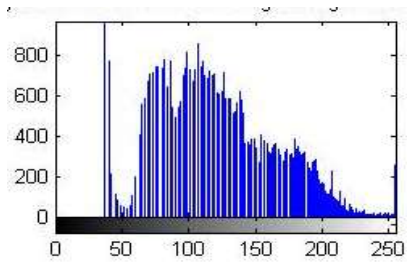

(q)

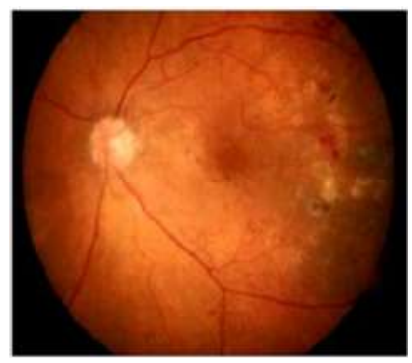

(t)

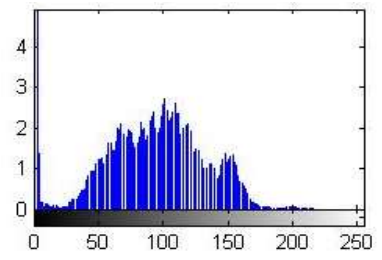

(w)

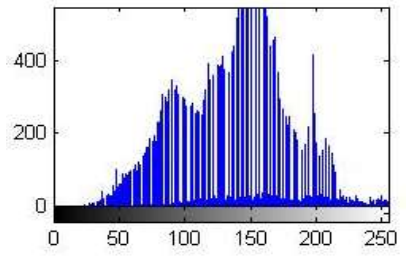

(1)

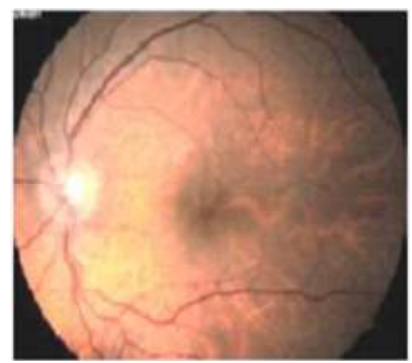

(o)

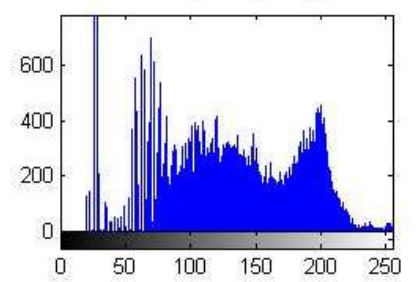

(r)

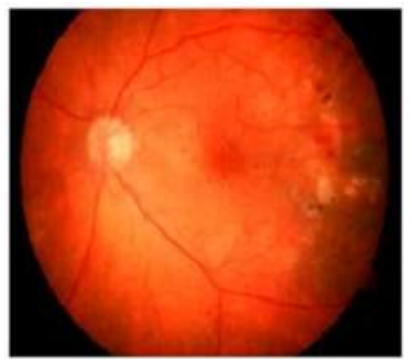

(u)

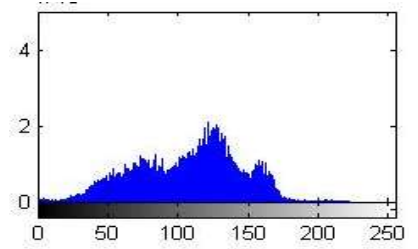

(x)

HE [7]

BCCE [8]

Proposed

Fig. 5: Comparison of results with respective histograms 


\section{REFERENCES}

[1] C. Reshmalakshmi and M. Sasikumar, "Image contrast enhancement using fuzzy technique", in Circuits, Power and Computing Technologies (ICCPCT), 2013 International Conference on, 2013, pp. 861-865.

[2] A. Gupta, A. Nag, J. Savani, and S. Natarajan, "Evolutionary approach and Spline Interpolation for contrast enhancement", in IEEE 2nd International Conference on Image Information Processing (ICIIP), 2013, pp. 181-186.

[3] Y. Wang, Q. Chen, and B. Zhang, "Image enhancement based on equal area dualistic sub-image histogram equalization method", IEEE Transactions on Consumer Electronics, vol. 15, pp. 68-75, FEBRUARY 1999.

[4] S. Kumar Maurya, P. Kumar Mishra, R. Kumar Singh, and A. Kumar Misra, "Image enhancement by spline interpolation and adaptive power spectrum cut-off of filtered images", in Advances in Engineering, Science and Management (ICAESM), 2012 International Conference on, 2012, pp. 423-428.

[5] T. M. Lehmann, C. Gonner, and K. Spitzer, "Survey: Interpolation Methods in Medical Image Processing", IEEE Transactions on Medical Imaging, vol. 18, pp. 1049-1075, NOV 1999.

[6] F.-C. Cheng and S.-C. Huang, "Efficient histogram modification using bilateral Bezier curve for the contrast enhancement", Journal of Display Technology, vol. 9, pp. 44-50, 2013.

[7] R. C. Gonzalez, R. E. Woods, and S. Eddins, "Digital Image Processing, 2002 by Prentice-Hall", Inc. Upper Saddle River, New Jersey, vol. 7458.

[8] S. J. Park and K. Krishna, "Method of Enhancing Contrast Using Bézier Curve", United States Patent, 2012.

[9] M. Mansourvar, M.A. Ismail, R.G. Raj, S.A. Kareem, S. Aik, R. Gunalan, et al. "The applicability of Greulich and Pyle atlas to assess skeletal age for four ethnic groups", Journal of forensic and legal medicine. vol. 22: pp. 26-29, 2014. pmid:24485416.

[10] M. Mansourvar, R.G. Raj, M.A. Ismail, S.A. Kareem, S. Shanmugam, W. Shahrom, et al. "Automated Web Based System For Bone Age Assessment Using Historam Technique", Malaysian Journal of Computer Science. vol. 25: pp.107, 2012.

[11] M. Mansourvar, M.A. Ismail, T. Herawan, R.G. Raj, S. Abdul Kareem, F.H. Nasaruddin, et al. "Automated Bone Age Assessment: Motivation, Taxonomies, and Challenges", Computational and mathematical methods in medicine. 2013.

[12] S. Mohan and M. Ravishankar, "Modified contrast limited adaptive histogram equalization based on local contrast enhancement for mammogram images", in Mobile Communication and Power Engineering, ed: Springer, 2013, pp. 397-403.

[13] V. Jaya and R. Gopikakumari, "IEM: a new image enhancement metric for contrast and sharpness measurements", Int. J. Comput. Appl, vol. 79, pp. 1-9, 2013.

[14] A. Masood and S. Ejaz, "An efficient algorithm for robust curve fitting using cubic Bezier curves", in Advanced Intelligent Computing Theories and Applications. With Aspects of Artificial Intelligence, 2010, pp. 255-262.

[15] Z. Habib, M. Sarfraz, and M. Sakai, "Rational cubic spline interpolation with shape control", Computers \& Graphics, vol. 29, pp. 594-605, 2005.

[16] K. Hirai, "Magnetic resonance imaging apparatus and contrast-enhanced image acquisition method", US20130241552 A1, 2011.

[17] Z. Lei, P. Sartor, Y. Inescu, and P. Wagner, "Contrast enhancement of digital images", US7102697 B2, 2006.

[18] K. Panetta, C. Gao, and S. Agaian, "No reference color image contrast and quality measures", IEEE Transactions on Consumer Electronics, vol. 59, pp. 643-651, 2013. 
[19] K. Yanai and K. Barnard, "Image region entropy: a measure of visualness of web images associated with one concept", in Proceedings of the 13th annual ACM international conference on Multimedia, 2005, pp. 419-422. 